CASE REPORT

\title{
Squamous cell carcinoma arising in a tall cell papillary carcinoma of the thyroid
}

\section{J Sutak, J S Armstrong, J E Rusby}

J Clin Pathol 2005;58:662-664. doi: 10.1136/icp.2004.021451

Transformation of differentiated thyroid cancer into poorly differentiated carcinoma is rare. This report describes a case in which preoperative fine needle aspiration suggested a squamous cell carcinoma whereas needle core biopsy favoured an undifferentiated carcinoma of probable thyroid origin. Histology of the subsequent total thyroidectomy specimen revealed a biphasic tumour comprising areas of tall cell papillary carcinoma merging with moderately to poorly differentiated squamous cell carcinoma. The immunohistochemical findings are discussed in detail.

A n 80 year old female patient presented with a three week history of a painless lump in her neck. She was a non-smoker, only occasionally drank alcohol, and denied difficulties with swallowing or breathing.

On examination she had a normal voice, was clinically euthyroid, and had a $1 \times 2 \mathrm{~cm}$, hard, non-tender lump to the left of the midline in her neck, which moved on swallowing and protrusion of the tongue. There was no associated lymphadenopathy and examination of the upper aerodigestive tract was normal.

An ultrasound showed a multinodular goitre with a prominent $2.5 \mathrm{~cm}$ nodule, which was largely solid, but contained a small cystic component in the isthmus of the thyroid gland. Fine needle aspiration cytology identified atypical, pleomorphic cells lying singly and in clusters. Some cells had keratinised cytoplasm, suggesting squamous cell carcinoma (fig l).

In view of this unusual cytology, an ultrasound guided core biopsy was performed to clarify the pathology before proceeding to surgery. This showed a poorly differentiated tumour with the suggestion of a papillary appearance.

Calcitonin measurement, 24 hour urinary hydroxymethylmandelic acid, and computed tomography of the chest and abdomen were normal.

At surgery, the mass lesion was palpable and the thyroid gland was tethered to muscle. There was associated oedema and palpable lymph nodes inferiorly on both sides. A total thyroidectomy was performed, skeletonising the trachea, preserving both recurrent laryngeal nerves and removing palpable nodes, but not as an en bloc resection. The patient made a good postoperative recovery, requiring calcium supplementation but with no voice changes.

\section{PATHOLOGY}

\section{Macroscopy}

The total thyroidectomy specimen weighed $31 \mathrm{~g}$ and measured $60 \times 50 \times 20 \mathrm{~mm}$.

The right lobe was enlarged and was almost completely replaced by a partially cystic, partially necrotic, white tumour,
$50 \times 30 \times 20 \mathrm{~mm}$. The isthmus and the left lobe also contained firm white areas. The tumour extended to the resection margin in several areas.

\section{Microscopy}

Microscopic examination revealed the diffuse involvement of the right lobe and the isthmus and partial involvement of the left lobe by a biphasic tumour. Areas showed a complex cystic pattern with delicate papillae containing fibrovascular cores. Some of the cysts were surrounded by a fibrous capsule showing focal calcification. The cells were columnar in shape, with abundant eosinophilic cytoplasm, and many cells were twice as long as they were wide. These tall cell features were present in over $50 \%$ of the tumour cells. The nuclei were round and some were optically clear or grooved. Merging with these areas there were irregular, infiltrating islands of moderately to poorly differentiated squamous cell carcinoma, embedded in desmoplastic stroma (fig 2). The squamous cells had a high nuclear to cytoplasmic ratio, hyperchromatic nuclei, prominent nucleoli, and eosinophilic cytoplasm. Focal keratinisation was observed.

The squamous component contained areas of necrosis and chronic inflammation. The tumour showed extracapsular spread with invasion into perithyroidal soft tissue reaching the surgical resection margin. The sampled skeletal muscle was uninvolved by the tumour. Two lymph nodes showed metastases of the squamous cell carcinoma component. The stage of the tumour was pT4NIbMx (TNM 2002).

Immunohistochemistry showed positivity for antibodies to pancytokeratin (AE1/3), CK7, and CK19 in both the papillary and the squamous cell component. Tumour cells of both components were negative for carcinoembryonic antigen and calcitonin.

Thyroid transcription factor 1 immunostaining showed strong, diffuse nuclear positivity in the papillary component

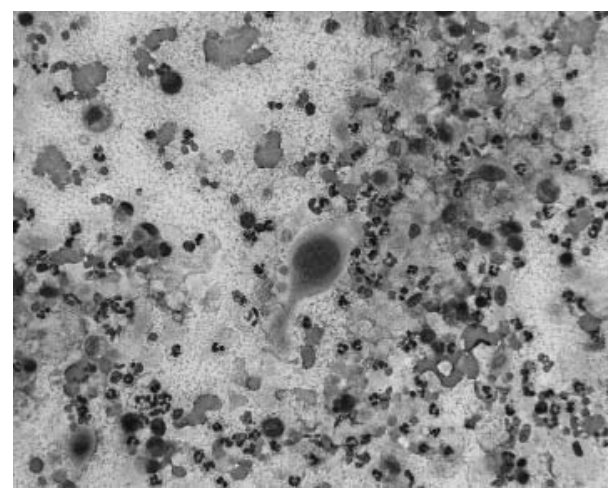

Figure 1 Fine needle aspiration cytology showed atypical, pleomorphic cells (Giemsa stain; original magnification, $\times 400$ ). 

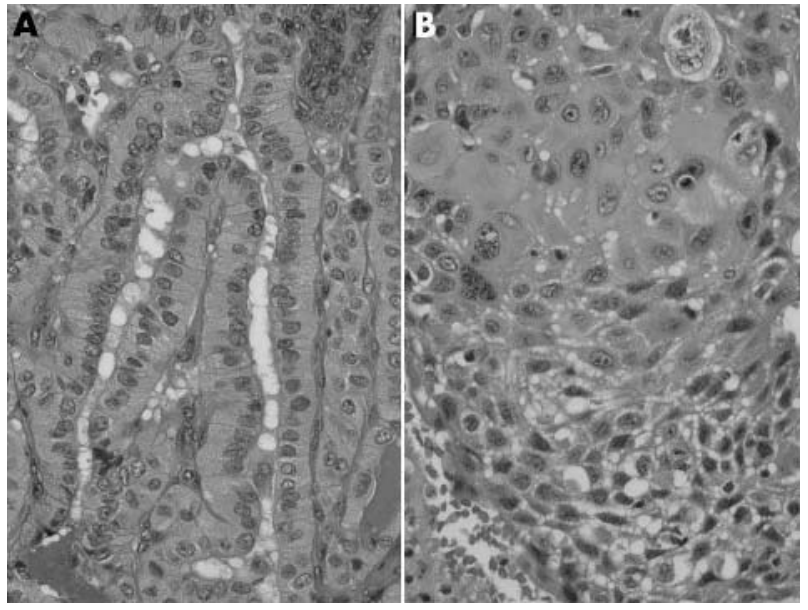

Figure 2 (A) Tall cell variant of papillary carcinoma and (B) moderately differentiated squamous cell carcinoma (haematoxylin and eosin stain; original magnification, $\times 400$ ).

but only weak, focal positivity in the squamous component. Thyroglobulin was positive in the papillary but negative in the squamous cell areas (fig 3 ).

A p53 stain showed strong and frequent nuclear positivity in the squamous cells but was completely negative in the papillary tumour cells (fig 4 ).

\section{DISCUSSION}

The tall cell variant of papillary carcinoma was described in 1976 by Hawk and Hazard, ${ }^{1}$ who identified this subtype in $10 \%$ of papillary thyroid cancers.

Histologically, the cells are columnar, twice as long as they are wide, with abundant eosinophilic cytoplasm. These are large tumours, occurring in older patients, and they often have an aggressive behaviour, with frequent capsular and vascular invasion, and tumour recurrence after surgical excision.

Squamous cells can be found in the thyroid as a result of persistence of thyroglossal ducts or structures derived from the branchial pouch, or as an expression of squamous metaplasia in Hashimoto's thyroiditis, papillary carcinoma, or other conditions.

Pure squamous cell carcinoma of the thyroid is exceptional. Most high grade thyroid tumours with squamous foci blend
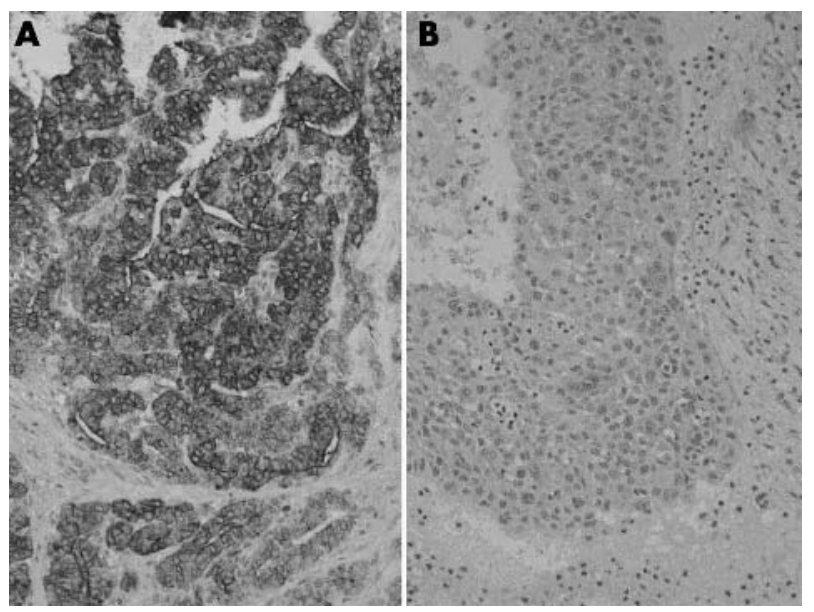

Figure 3 Thyroglobulin immunostaining showing (A) strong positivity in the papillary area, but (B) negativity in the squamous cell area (antithyroglobulin; original magnification, $\times 200$ ).

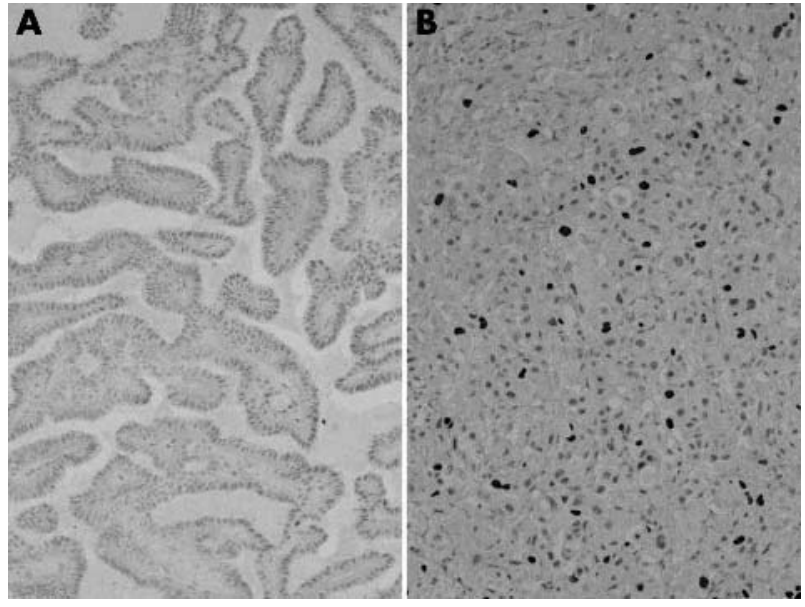

Figure 4 p53 immunostaining was (A) negative in the papillary tumour cells, but (B) showed frequent nuclear positivity in the squamous cells (anti-p53; original magnification, $\times 200$ ).

\section{Take home messages}

- We describe a rare case of transformation of papillary tall cell carcinoma of the thyroid into moderately to poorly differentiated squamous cell carcinoma

- Histology of the subsequent total thyroidectomy specimen revealed a biphasic tumour comprising areas of tall cell papillary carcinoma merging with moderately to poorly differentiated squamous cell carcinoma

- Such a transformation is rare and usually confers a poor prognosis

with areas of undifferentiated carcinoma and are generally placed in the latter category because of their similar natural history.

In the presence of squamous cell carcinoma involving the thyroid, the possibility should also be considered of secondary direct involvement from tumour of the larynx or trachea, or a metastasis from lung or other sites.

Coexistence of tall cell papillary carcinoma with squamous cell carcinoma was described, implying transformation of the former to the latter in a fashion analogous to that of undifferentiated carcinoma. It has been suggested that the tall cell variant of papillary carcinoma has a particular tendency to evolve into the spindle cell type of squamous cell carcinoma. $^{23}$

This transformation is rare and usually confers a poor prognosis, and despite current therapeutic methods the survival rate is very low..$^{2-6}$

This particular case report describes the histological and immunohistochemical findings of this rare transformation of papillary tall cell carcinoma of the thyroid into moderately to poorly differentiated squamous cell carcinoma.

\section{ACKNOWLEDGEMENTS}

We would like to thank Dr E Sheffield for his second opinion on this case.

\section{Authors' affiliations}

J Sutak, J S Armstrong, Department of Histopathology, Great Western Hospital, Swindon and Marlborough NHS Trust, Marlborough Road, Swindon SN3 6BB, UK 
J E Rusby, Department of General Surgery, Great Western Hospital, Swindon and Marlborough NHS Trust

Correspondence to: Dr J Armstrong, Department of Pathology, Great Western Hospital, Swindon and Marlborough NHS Trust, Marlborough Road, Swindon SN3 6BB, UK; Janette.Armstrong@smnhst.swest.nhs.uk

Accepted for publication 22 November 2004

\section{REFERENCES}

1 Hawk WA, Hazard JB. The many appearances of papillary carcinoma of the thyroid. Cleve Clin Q 1976;43:207-15.
2 Bronner MP, LiVolsi VA. Spindle cell squamous carcinoma of the thyroid: an unusual anaplastic tumor associated with tall cell papillary cancer. Mod Pathol 1999:4:637-43.

3 Saunders CA, Nayar R. Anaplastic spindle-cell squamous carcinoma arising in association with tall-cell papillary cancer of the thyroid: a potential piffall. Diagn Cytopathol 1999;21:413-18.

4 Kleer CG, Giordano T, Merino MJ. Squamous cell carcinoma of the thyroid: an aggressive tumor associated with tall cell variant of papillary thyroid carcinoma. Mod Pathol 2000;13:742-6.

5 Pitman KT, Johnson JT. Papillary thyroid carcinoma associated with squamous cell carcinoma of the head and neck: significance and treatment.

Am J Otolaryngol 1996;17:190-6.

6 Kebapci N, Efe B, Kabukcuoglu S, et al. Diffuse sclerosing variant of papillary thyroid carcinoma with papillary squamous cell carcinoma. J Endocr Invest 2002;25:730-4. 